\title{
Web Stream Processing Workshop Chairs' Welcome
}

Applications in different domains require reactive processing of massive, dynamically generated streams of data. This trend is increasingly visible also on the Web, where more and more streaming sources are becoming available. These originate from social networks, sensor networks, the Internet of Things (IoT) and many other technologies that use the Web as a platform for sharing data. This has resulted in new Web-centric efforts such as the Web of Things (WoT), which focuses on exposing and describing the IoT resources on the Web; or the Social Web which provides protocols, vocabularies, and APIs to facilitate access to social communications and interactions on the Web.

It is our great pleasure to welcome you to the WWW 2018 Web Stream Processing Workshop. We received seven proposals from all around the world covering a broad range of topics, from RDF Stream Processing to Stream Reasoning, from IoT to Deep Learning. The review process led to the acceptance of five talks:

- A preliminary Open Data publishing strategy for real-time data in Flanders

- Adaptive Optimisation for Continuous Multi-way Joins over RDF Streams

- Analysis of Sub-Topic Discovery Algorithms for Real-Time Information Summarization

- Personalized Real-Time Monitoring of Amateur Cyclists on Low-End Devices: Proof-ofConcept \& Performance Evaluation

- Spatio-Temporal Analysis for Smart City Data

These contributions include both theoretical and practical developments in the area, spanning from streaming data analytics and monitoring in smart cities, to techniques for query optimization and information summarization for dynamic data streams. In all these scenarios, the Web dimension brings an additional challenge, given the large degree of heterogeneity and decentralization found in these environments. Furthermore, the accepted talks provide address distinct problems that offer different perspectives, thus enriching the debate and discussions expected during the workshop.

We are sure that the talks are valuable resources to develop flexible and scalable analytical processing for streaming data on the Web, enabling decentralized processing, publication and discovery of streaming data, as well as integration mechanisms at Web scale.

\author{
Payam Barnaghi Jean-Paul Callbimonte \\ Workshop chair Workshop Chair \\ University of Surrey University of Applied \\ Daniele Dell'Aglio \\ Workshop Chair \\ University of Zurich \\ Switzerland HES-SO
}

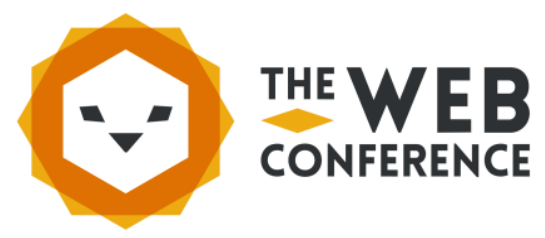




\section{Web Stream Processing Workshop Organization}

Workshop Chairs: Payam Barnaghi (University of Surrey, United Kingdom)

Jean-Paul Calbimonte (University of Applied Sciences and Arts Western

Switzerland HES-SO, Switzerland)

Daniele Dell'Aglio (University of Zurich, Switzerland)

Session Chairs: Maria Edo (University of Granada, Spain)

Andrea Mauri (Delft University of Technology, Netherlands)

Program Committee: Muhammad Intizar Ali (Insight Centre for Data Analytics, National University of Ireland, Galway, Ireland)

Shirin Enshaeifar (University of Surrey, United Kingdom)

Javier D. Fernández (Vienna University of Economics and Business, Austria)

Alasdair Gray (Heriot-Watt University, United Kingdom)

Tobias Grubenmann (University of Zurich, Switzerland)

Robin Keskisärkkä (Linköping University, Sweden)

Alessandro Margara (Politecnico di Milano, Italy)

Andrea Mauri (Delft University of Technology, Netherlands)

Josiane Xavier Parreira (Siemens AG, Austria)

Danh Le Phuoc (TU Berlin, Germany) 